Faculty of Science

Faculty Publications

This is a post-review version of the following article:

A microfluidic approach for high-throughput droplet interface bilayer (DIB)

formation

C.E. Stanley, K.S. Elvira, X.Z. Niu, A.D. Gee, O. Ces, J.B. Edel and A.J. deMello

2010

The final published version of this article can be found at:

https://doi.org/10.1039/B924897H

Citation for this paper:

Stanley, C.E., Elvira, K.S., Niu, X.Z., Gee, A.D., Ces, O., Edel, J.B. \& deMello, A.J. (2010). A microfluidic approach for high-throughput droplet interface bilayer (DIB) formation. Chemical Communications, 46(10), 1620-1622. 


\title{
A microfluidic approach for high-throughput droplet interface bilayer (DIB) formation $\uparrow$
}

\author{
C.E. Stanley ${ }^{\ddagger a, b}$ K.S. Elvira, ${ }^{\ddagger a, c}$ X.N. Niu, ${ }^{a, c}$ A.D. Gee, ${ }^{d}$ O. Ces, ${ }^{* a, b}$ J.B. Edel ${ }^{* a, c}$ and A.J. deMello ${ }^{* a}$ \\ Received (in XXX, XXX) Xth XXXXXXXXX 200X, Accepted Xth XXXXXXXXX 200X \\ ${ }_{5}$ First published on the web $X t h X X X X X X X X X 200 X$ \\ DOI: 10.1039/b000000x
}

We present a simple, automated method for high-throughput formation of droplet interface bilayers (DIBs) in a microfluidic device. We can form complex DIB networks that are able to fill ${ }_{10}$ predefined three dimensional architectures. Moreover, we demonstrate the flexibility of the system by using a variety of lipids including 1,2-diphytanoyl-sn-glycero-3-phosphocholine (DPhPC) and 1,2-dioleoyl-sn-glycero-3-phosphocholine (DOPC).

Cell membranes are ubiquitous in living organisms, composed 15 predominantly of a bilayer containing amphiphilic lipid molecules and proteins. Not only do they act as a barrier which separates the intra- and intercellular milieu, but they also play a central role in choreographing cellular tasks. It is therefore unsurprising that a wide variety of platforms are 20 being developed for the formation of artificial lipid bilayers, where studies of a biophysical nature can be conducted to probe questions on protein and lipid function. ${ }^{1}$

Traditional methods for the formation of artificial planar bilayers, or black lipid membranes (BLMs), include the 25 Montal-Mueller method. ${ }^{2}$ In this system a Teflon film containing an aperture is pulled through a Langmuir-Blodgett lipid film, ${ }^{3}$ where two lipid monolayers are brought into contact thus resulting in bilayer formation. There are, however, major problems associated with such techniques: 30 both bilayer stability and reproducibility are poor. Hence recent years have seen a wealth of research centred upon droplet interface bilayers (DIBs), ${ }^{4}$ a methodology first introduced by Funakoshi $^{5}$ and Holden. ${ }^{6}$ This approach involves bringing together two or more aqueous droplets 35 submerged in an oil environment, where the aqueous-oil interface is stabilised with a monolayer of amphiphilic lipid molecules. Bilayer formation occurs at the point of contact and can be achieved either by doping the lipid into the aqueous ('lipid-in') or oil ('lipid-out”) phase. ${ }^{7}$

40 Several advances have been made with respect to enhancing the screening capability of the DIB platform following the first version presented in 2006, where

${ }^{a}$ Department of Chemistry, Imperial College London, Exhibition Road, 45 South Kensington, London, UK SW7 $2 A Z$.

${ }^{b}$ Chemical Biology Centre, Imperial College London, Exhibition Road, South Kensington, London, UK SW7 $2 A Z$.

${ }^{c}$ Institute of Biomedical Engineering, Imperial College London,

Exhibition Road, South Kensington, London, UK SW7 $2 A Z$.

$5^{50}{ }^{d}$ GSK, Clinical Imaging Centre, Imperial College London, Hammersmith Hospital, Du Cane Road, London, UK W12 ONN.

$\uparrow$ Electronic Supplementary Information (ESI) available: detailed experimental procedures and movies 1-4. See DOI: 10.1039/b000000x/

$\$$ These authors contributed equally to this work.
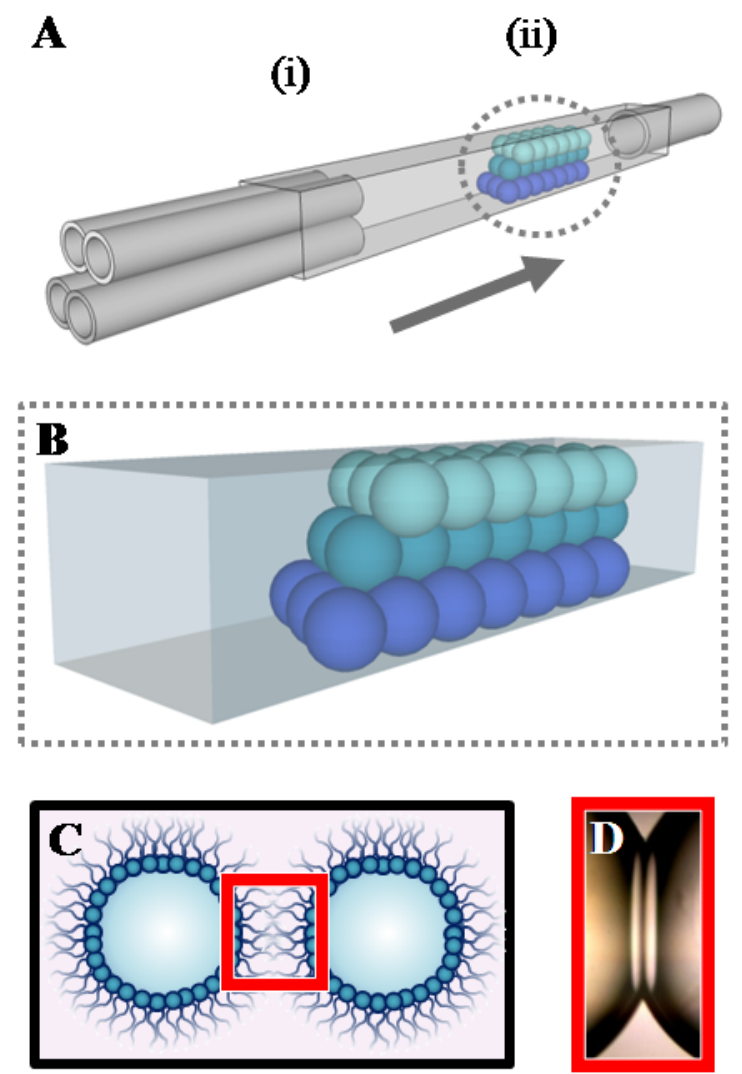

55 Fig. 1 A) Schematic of the experimental set-up. Water-in-oil droplets were pumped into PTFE (polytetrafluoroethylene) tubing (inner diameter $100 \mu \mathrm{m}$, note four pieces were used to create uniform flow through the channel) and introduced into the device at point (i). Droplet interface bilayer (DIB) formation in a high-throughput manner is observed 60 downstream at (ii). Arrow indicates direction of flow through the device. B) Example of droplets stacking in three dimensions to fill the channel. C) Pictorial representation of water-in-oil droplets surrounded by a lipid monolayer; DIB formation occurs when the two monolayers come into contact with one another (highlighted by a red box). D) Bright field 65 microscopy image illustrating a typical DIB achieved in our experiments; the characteristic shadowing indicates successful DIB formation (see also Movie 1, ESI, for an example of DIB formation). Figure not to scale.

mechanical pumping within a microfluidic device was employed for bilayer assembly. ${ }^{5,8}$ Elegant work by the Bayley 70 group has yielded DIB networks comprised of tens of droplets; $;, 9$ more recently the same group has demonstrated the ability of such networks to process electrical inputs, ${ }^{10}$ by incorporation of engineered staphlococcal $\alpha$-haemolysin pores. Electrical methods for droplet manipulation via 
A
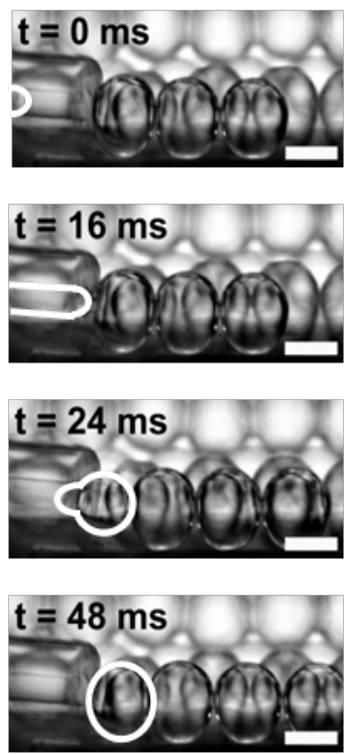

B
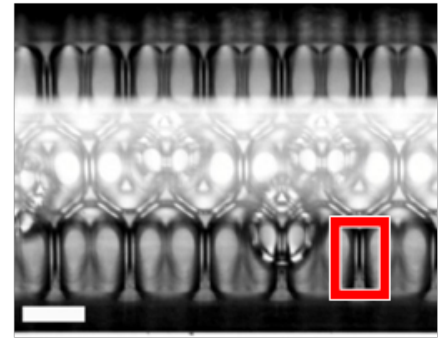

C

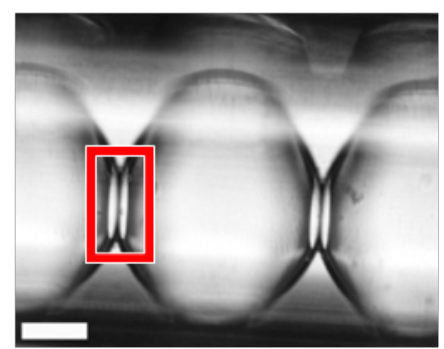

Fig. 2 A) Series of time frames illustrating formation of water-in-oil droplets containing vesicles comprised of DPhPC $(2 \mathrm{mg} / \mathrm{ml})$. Droplets are formed (stages of droplet formation are highlighted in white) and carried 5 in PTFE tubing (inner diameter $=100 \mu \mathrm{m}$ ) to the point at which they are released into the device. The tubing can be seen on the lower left hand corner of the figures in A. Droplets are small and therefore stack in three dimensions upon entering the larger glass channel. Images B) and C) demonstrate the extensive networks of DIBs that are formed in the 10 channel downstream (red boxes denote DIBs). Images show a top view of DIB networks in the channel (in B, droplets stack in three dimensions, as shown in the pictorial representation in Fig. 1B). Note the tunable droplet size due to the different volumetric flow rates, which are $3 \mu \mathrm{l} / \mathrm{min}$ and $6 \mu \mathrm{l} / \mathrm{min}$ for $\mathrm{B}$ and $\mathrm{C}$ respectively. Lighter area at the centre of B and the 15 droplet asymmetry suggested in $\mathrm{C}$ are effects caused by diffraction of light in the square channel. Scale bar $=200 \mu \mathrm{m}$.

dielectrophoresis (DEP) $)^{11}$ and electrowetting on dielectric (EWOD) $)^{12}$ are also being utilised, although a linear three-drop sequence has been the most complex setup reported to date.

20 More high-throughput methods for the measurement of transmembrane ion currents do exist, where the bilayer is vertically orientated. ${ }^{13}$ In these systems a more classical BLM type of approach, ${ }^{14,15}$ or the use of gravity, ${ }^{16}$ have been employed to deposit DIBs. Again these systems only possess

25 one bilayer per experimental unit and often involve complex device design. Clearly, there is a great need in the field for a method able to produce extensive networks of DIBs that does not depend upon manual management.

Herein we describe a system for the automated formation of ${ }_{30}$ DIBs in a high-throughput manner using a simple microfluidic device (Fig. 1). Moreover, we are able to form complex networks of DIBs in three dimensions and fill chambers of predefined size and shape. Furthermore, our platform possesses the configurational flexibility to form droplets in 35 linear sequences of alternating composition. To the best of our knowledge, this has not been achieved to date, and represents a large contribution towards the creation of a platform for complex DIB network formation.

Water-in-oil microdroplets ${ }^{17,18}$ were formed using a 40 microfluidic platform. A syringe pump working in refill mode

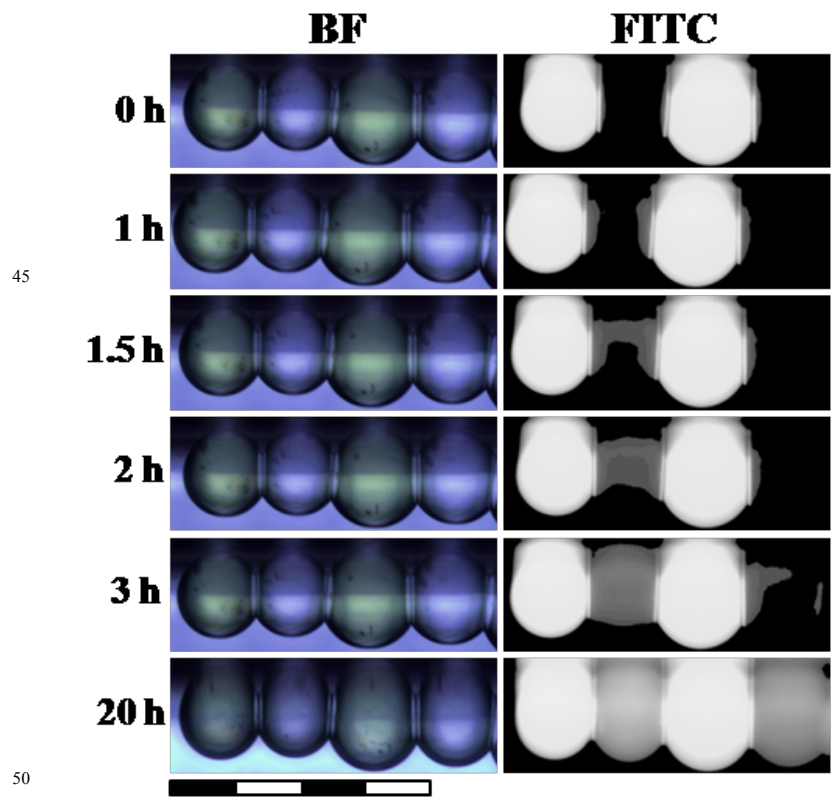

Fig. 3 Images showing leakage of fluorescein across DIBs. Droplets are in an $\mathrm{ABABB}$ formation (where $\mathrm{A}$ and $\mathrm{B}$ are fluorescent and nonfluorescent droplets respectively. Note that the fifth droplet is off camera). The slight variation in droplet size is due to an artifact of the tubing at the 55 droplet formation site. The left hand column shows bright field (BF) images; the corresponding fluorescence microscopy images taken using a fluorescein isothiocyanate (FITC) filter can be observed in the right hand column. Images were captured at $0 \mathrm{~h}, 1 \mathrm{~h}, 1.5 \mathrm{~h}, 2 \mathrm{~h}, 3 \mathrm{~h}$ and $20 \mathrm{~h}$. DIBs are composed of DOPC and fluorescent droplets contained $250 \mu \mathrm{M}$ 60 fluorescein. Droplets $(\sim 35 \mathrm{nl}, 400 \mu \mathrm{m}$ diameter $)$ were formed with a volumetric flow rate of $12 \mu \mathrm{l} / \mathrm{min}$. When using DOPC, higher flow rates were needed to create droplets of intermediate size; this is likely to be due to differences in the interfacial tension or viscosities of the aqueous phase. Scale bar $=1600 \mu \mathrm{m}$ (total length).

65 was used to aspirate liquid via the thin PTFE tubing (Fig. 1A). A two channel autosampler was used to move the tip of the tubing into oil and vesicle solutions alternately, thereby creating vesicle droplets and pumping them into a glass channel. DIB networks are then produced and can be observed at a point downstream of 70 droplet formation (Fig. 1A). Droplet composition is described in detail in the ESI; it suffices to say here that they contain pre-prepared vesicles $c a .100 \mathrm{~nm}$ in diameter. When the aqueous lipid solution is used to make droplets in hexadecane oil, the lipids re-orientate themselves to form a monolayer at 75 the oil-aqueous interface due to favourable interactions between the hydrophobic lipid tails and the oil. When the droplets come into contact, a droplet interface bilayer is formed between them (Fig. 1B and C). Interestingly, previous work published on DIB formation has focussed on DPhPC. In 80 this work we demonstrate DIB formation with a more biologically relevant lipid, DOPC.

Droplets formed in this manner have a size that can be controlled by the volumetric flow rate, with a typical size variation of only $3.8 \%$. When incorporating vesicles 85 composed of the lipid DPhPC into the aqueous phase, small droplets $(\sim 5 \mathrm{nl}, 200 \mu \mathrm{m}$ diameter $)$ are formed at low volumetric flow rates $(3 \mu \mathrm{l} / \mathrm{min})$, whilst larger droplets $(\sim 100 \mathrm{nl}, 600 \mu \mathrm{m}$ diameter) are formed at higher flow rates (6 $\mu \mathrm{l} / \mathrm{min}$ ) but at a lower frequency (Fig $2 \mathrm{~B}$ and $\mathrm{C}$ 90 respectively). The variation in droplet size affords a variation 
in the surface area of the DIBs formed per droplet. It can be observed that small droplets stack neatly in three dimensions filling the chamber (see Movie 3, ESI), therefore forming DIBs with all neighbouring droplets. Large droplets merely 5 participate in the formation of two bilayers, as they stack in a linear dimension only due to space constraints (see Movie 2, ESI).

The small size of the input tubing (when compared to the glass microchannel) allows formation of droplets which are 10 relatively small. This is important because the droplets do not completely fill the glass channel, therefore adopting a spherical shape due to surface tension. Conversely, if the dimensions of the droplets are comparable with the channels, they will adopt a plug or cigar-like shape. In this situation 15 droplet merging can be induced as the lipid membrane is stretched upon droplet-channel interaction. Therefore our device creates an ideal environment for DIB formation, where the droplets are brought together gently, whilst still allowing sufficient time for bilayer formation.

20 Confirmation of successful DIB formation (and the concurrent exclusion of any residual oil) is achieved using optical measurements. This method involves a fluorescence leakage assay performed with the dye fluorescein. It has been reported that fluorescein successfully leaks across DOPC 25 bilayers, in contrast to other fluorophores such as carboxyfluorescein. ${ }^{19}$ This is attributed to the fact that fluorescein lacks an additional carboxylic acid group, hence rendering it more soluble in the bilayer.

Sequential ABAB droplets were formed between 30 fluorescent and non-fluorescent droplets (where A contains $250 \mu \mathrm{M}$ fluorescein and B denotes a non-fluorescent droplet) and were inserted into the microfluidic device (Fig. 3). Initially there is no leakage of fluorescein. However, leakage does occur over time, and is first visible after 60 minutes. It is 35 important to note that droplets in this experiment are configured in an ABABB pattern. This asymmetry provides two different environments for the two non-fluorescent droplets displayed in Fig. 3. In the first case, two fluorescent droplets will leak into one non-fluorescent droplet, whereas in 40 the second case, only one fluorescent droplet will allow leakage into the non-fluorescent drop. The asymmetry will affect the amount of leakage observed. Control experiments (not shown) were performed to confirm that there is no leakage of fluorescein into the surrounding hexadecane oil. ${ }_{45}$ Equal volumes of the aqueous fluorescent phase and hexadecane were stored in the same vial for five days. Leakage into the oil was monitored by fluorescence spectroscopy, with no discernible increase in fluorescence emission observed at $520 \mathrm{~nm}$.

50 ABAB droplet formation is of great relevance to DIB research, as it enables facile formation of asymmetric bilayers. With this system we are able to form AAAA, BBBB and $\mathrm{ABAB}$ sequences, but this can easily be extended to networks with three or more different kinds of droplets (such 55 as $\mathrm{ABCABC}$ ) thus enabling the creation of complex combinations of linear asymmetric bilayers. Moreover, we can create three dimensional networks in the described device (Fig. 2B) to create what Maglia et al. call 'systems with higher-level properties'. ${ }^{10}$

60 In conclusion, this work introduces several new advances for generating DIB platforms, increasing their potential impact in areas such as drug discovery by allowing extensive networks of DIBs to be formed automatically and recognised with ease. We foresee future developments to couple this ${ }_{65}$ platform technology with increasingly complex microfluidic chips, which can be filled with DIBs in high-throughput, whilst simultanouesly varying the sequential identity and size of the input droplets. Furthermore, variations in chamber geometry, as well as droplet dimensions (by varying the 70 volumetric flow rate), can afford DIBs that differ both in size and hence membrane stress.

This work was supported in part by the EPSRC (EP/G00465X/1), RCUK (EP/D048664/1) and GSK.

\section{Notes and references}

751 R. Phillips, T. Ursell, P. Wiggins and P. Sens, Nature, 2009, 459, 379-385.

2 M. Montal and P. Mueller, Proc. Natl. Acad. Sci. U. S. A, 1972, 69, 3561-3566.

3 K. B. Blodgett, J. Am. Chem. Soc., 1935, 57, 1007-1022.

804 H. Bayley, B. Cronin, A. Heron, M. A. Holden, W. L. Hwang, R. Syeda, J. Thompson and M. Wallace, Mol. BioSyst., 2008, 4, 11911208.

5 K. Funakoshi, H. Suzuki and S. Takeuchi, Anal. Chem., 2006, 78, 8169-8174.

856 M. A. Holden, D. Needham and H. Bayley, J. Am. Chem. Soc., 2007, 129, 8650-8655.

7 W. L. Hwang, M. Chen, B. Cronin, M. A. Holden and H. Bayley, $J$. Am. Chem. Soc., 2008, 130, 5878-5879.

8 N. Malmstadt, M. A. Nash, R. F. Purnell and J. J. Schmidt, Nano 90 Lett., 2006, 6, 1961-1965.

9 W. L. Hwang, M. A. Holden, S. White and H. Bayley, J. Am. Chem. Soc., 2007, 129, 11854-11864.

10 G. Maglia, A. J. Heron, W. L. Hwang, M. A. Holden, E. Mikhailova, Q. Li, S. Cheley and H. Bayley, Nat. Nanotechnol., 2009, 4, 437-440.

9511 S. Aghdaei, M. E. Sandison, M. Zagnoni, N. G. Green and H. Morgan, Lab Chip, 2008, 8, 1617-1620.

12 J. L. Poulos, W. C. Nelson, T.-J. Jeon, C.-J. Kim and J. J. Schmidt, Appl. Phys. Lett., 2009, 95, 013706.

13 R. Syeda, M. A. Holden, W. L. Hwang and H. Bayley, J. Am. Chem. 100 Soc., 2008, 130, 15543-15548.

14 B. LePioufle, H. Suzuki, K. V. Tabata, H. Noji and S. Takeuchi, Anal. Chem., 2008, 80, 328-332.

15 M. Zagnoni, M. E. Sandison and H. Morgan, Biosens. Bioelectron., 2009, 24, 1235-1240.

10516 J. L. Poulos, T.-J. Jeon, R. Damoiseaux, E. J. Gillespie, K. A. Bradley and J. J. Schmidt, Biosens. Bioelectron., 2009, 24, 18061810.

17 X. Niu, S. Gulati, J. B. Edel and A. J. deMello, Lab Chip, 2008, 8, $1837-1841$.

11018 A. Huebner, S. Sharma, M. Srisa-Art, F. Hollfelder, J. B. Edel and A. J. deMello, Lab Chip, 2008, 8, 1244-1254.

19 J. N. Weinstein, R. Blumenthal and R. D. Klausner, Method. Enzymol., 1986, 128, 657-668. 